\title{
Pancreatic Intraductal Papillary Mucinous Neoplasm with Hyaline Globules (Thanatosomes): Report of Two Cases
}

\author{
Mitsuhiro Tachibana (1D \\ Ryohei Koreyasu ${ }^{2}$ \\ Kazuyasu Kamimura ${ }^{2}$ \\ Yutaka Tsutsumi ${ }^{1,3}$ \\ 'Department of Diagnostic Pathology, \\ Shimada City General Medical Center, \\ Shimada, Shizuoka, Japan; ${ }^{2}$ Department of \\ Surgery, Shimada City General Medical \\ Center, Shimada, Shizuoka, Japan; \\ ${ }^{3}$ Diagnostic Pathology Clinic, Pathos \\ Tsutsumi, Inazawa, Aichi, Japan
}

Correspondence: Mitsuhiro Tachibana Department of Diagnostic Pathology, Shimada City General Medical Center, Noda 1200-5, Shimada, Shizuoka, 427-8502, Japan

Tel +8I-547-35-2III (ext. 2273)

Fax +8I-547-35-2I82

Email 0206mtachi@gmail.com

\begin{abstract}
Hyaline globules (HGs; thanatosomes) represent a morphologic entity representing a metabolic imbalance common to all cell types. HGs, intracytoplasmic eosinophilic globular accumulations of proteinaceous material of varying sizes, have been observed in varied tumors and benign tissues. Different explanations have been proposed for their formation, according to the tumor type and anatomic location. An earlier study suggested that HGs were closely related to apoptosis. There are some reports describing HGs in pancreatic neoplasms, such as intraductal oncocytic papillary neoplasm, solidpseudopapillary neoplasm, oncocytic endocrine neoplasm, and invasive ductal adenocarcinoma; however, this is the first report describing HGs in pancreatic intraductal papillary mucinous neoplasm (IPMN). An ultrastructural study was performed to visualize HGs in two pancreatic IPMNs of gastric type (one non-invasive malignancy and another adenoma). Light microscopically, intracytoplasmic HGs were clustered multifocally. HGs were periodic acid-Schiff-positive and diastase-resistant, and fuchsinophilic with Masson's trichrome stain. The diameter ranged from 4.7 to $20.6 \mu \mathrm{m}$ (mean: 13.3, median: 14.1). They were mainly seen at the supranuclear position and occasionally with subnuclear location. Ultrastructurally, HGs were round in shape and homogenously electrondense without mitochondria or chromatin-like condensation. The nuclei of HGscontaining mucous columnar cells appeared intact without evidence of apoptosis. It is worth emphasizing that HGs in the pancreatic IPMN of gastric type belong not to apoptotic bodies but to proteinaceous secretory materials.
\end{abstract}

Keywords: pancreas, pancreatic intraductal papillary mucinous neoplasm, hyaline globules, thanatosomes

\section{Introduction}

Hyaline globules (HGs), also termed as thanatosomes, have been identified in various types of cells and tissues, and described in the normal, subnormal, nonneoplastic and neoplastic conditions. ${ }^{1}$ HGs represent a well-described morphologic and functional entity that may serve as a microscopic hallmark of cellular injury and apoptosis. ${ }^{1,2}$ There are some reports describing HGs in pancreatic neoplasms, such as intraductal oncocytic papillary neoplasm, solid-pseudopapillary neoplasm, oncocytic endocrine neoplasm, and ductal adenocarcinoma. ${ }^{3-5}$ Herein, we report two cases of gastric type intraductal papillary mucinous neoplasm (IPMN) of the pancreas, both lesions (one non-invasive malignancy and another adenoma) being rich in HGs, and ultrastructural evaluation of HGs is shown. 


\section{Case Presentation}

\section{Case I}

\section{Clinical Summary}

A 76-year-old Japanese woman nonsmoker was referred to Shimada Municipal Hospital, Shimada, Shizuoka, Japan, because of bilateral lower abdominal pain lasting for two years. The serum level of carcinoembryonic antigen was $7.8 \mathrm{ng} / \mathrm{mL}$ (reference value $<5.0$ ), with that of carbohydrate antigen 19-9 (CA19-9) being 51.6 U/mL (reference value $<37$ ). Endoscopic ultrasound study, abdominal computed tomography and magnetic resonance cholangiopancreatography revealed a $30 \mathrm{~mm}$ sized IPMN of main pancreatic duct origin with a small mural nodule at the pancreatic head. Neither lymphadenopathy nor metastatic lesion was noted. Stomach-preserving pancreatoduodenectomy was performed.

\section{Gross, Light Microscopic, Histochemical and Immunohistochemical Analysis}

Grossly, a mucinous cystic lesion measured $72 \mathrm{~mm}$, and a $10 \mathrm{~mm}$-sized mural nodule was attached (Figure 1A). The cystic tumor was demarcated and encapsulated. In hematoxylin and eosin (H\&E)-stained preparations, papillary/villous growth of tall columnar cells was observed in the dilated pancreatic duct. There were nuclear pleomorphism, increased mitosis and stratification of the nuclei, indicating a malignant potential (Figure 1B). No invasive growth was identified histologically. According to the World Health Organization (WHO) Classification of Tumours of the Digestive System, the diagnosis should be intraductal papillary-mucinous carcinoma, noninvasive. The surrounding non-neoplastic pancreas revealed no significant changes. The tumor cells expressed gastric-type mucin immunohistochemically: mucin 1 (MUC1)-, MUC2-, MUC5AC+ (Figure 1C), and MUC6 focally + (Figure 1D). Caudal-type homeobox-2 (CDX2) was negative. Characteristically, intracytoplasmic eosinophilic hyaline globules (HGs) were multifocally clustered (Figure 1E). The inclusions were mainly seen at the supranuclear location, and occasionally with a subnuclear pattern. The size ranged from 4.7 to $14.5 \mu$ mean: 9.1, median: 8.1). The HGs were stained with periodic acidSchiff (PAS) reaction with varying degrees of intensity (from light pink to bright red in color). The PAS reactivity was diastase-resistant. With Masson's trichrome, the HGs showed bright red staining with fuchsinophilia (Figure 1F). Immunohistochemically, the HGs were negative for
MUC5AC, MUC6 (Figure 1G), p53, ubiquitin (Figure 1H), 1-antitrypsin (Figure 1I), and cleaved caspase-3 (Figure 1J).

\section{Follow-Up Information}

Distant metastasis was not seen. No recurrence was noted clinically for the following four years after surgery.

\section{Case 2}

\section{Clinical Summary}

A 60-year-old Japanese woman nonsmoker was referred to Shimada Municipal Hospital for the checkups of her diabetes mellitus. Abdominal computed tomography revealed an $8 \mathrm{~mm}$-sized IPMN of pancreatic branch duct type with an intraductal nodule at the pancreatic hook. The serum level of CA19-9 was $43.4 \mathrm{U} / \mathrm{mL}$. Neither lymphadenopathy nor metastatic lesion was noted. Stomach-preserving pancreatoduodenectomy was performed.

\section{Gross, Light Microscopic, Histochemical and Immunohistochemical Analysis}

Grossly, a mucinous lesion, $45 \mathrm{~mm}$ in size, was demarcated and encapsulated in the pancreatic parenchyma (Figure 2A). Light microscopically, the pancreatic hook contained an IPMN of benign adenoma type growing in the pancreatic duct (intraductal papillary-mucinous adenoma, according to the WHO Classification). The surrounding non-neoplastic pancreas revealed no significant changes. Intracytoplasmic HGs (thanatosomes) were multifocally clustered in the supranuclear cytoplasm of the adenoma cells (Figure 2B). Their size ranged from 11.5 to $21.6 \mu \mathrm{m}$ (mean: 17.5 , median: 17.0 ). PAS reactivity and fuchsinophilia of the HGs were confirmed. Immunohistochemically, gastric-type mucin was observed [MUC1-, MUC2-, MUC5AC+ (Figure 2c), and MUC6+ (Figure 2D)]. CDX2 was not expressed. The HGs were negative for MUC5AC, MUC6 (Figure 2E), p53, ubiquitin (Figure 2F), 1-antitrypsin (Figure 2G), and cleaved caspase-3 (Figure 2H).

\section{Follow-Up Information}

The patient is currently doing well, 13 months after surgery.

\section{Ultrastructural Studies}

The surgical specimens were fixed in phosphate-buffered $10 \%$ formalin, $\mathrm{pH} 7.4$ (Kanto Chemical, Tokyo), and embedded in paraffin (Parabett 60 GR, Muto Pure Chemicals, Tokyo). The routinely prepared paraffin blocks 


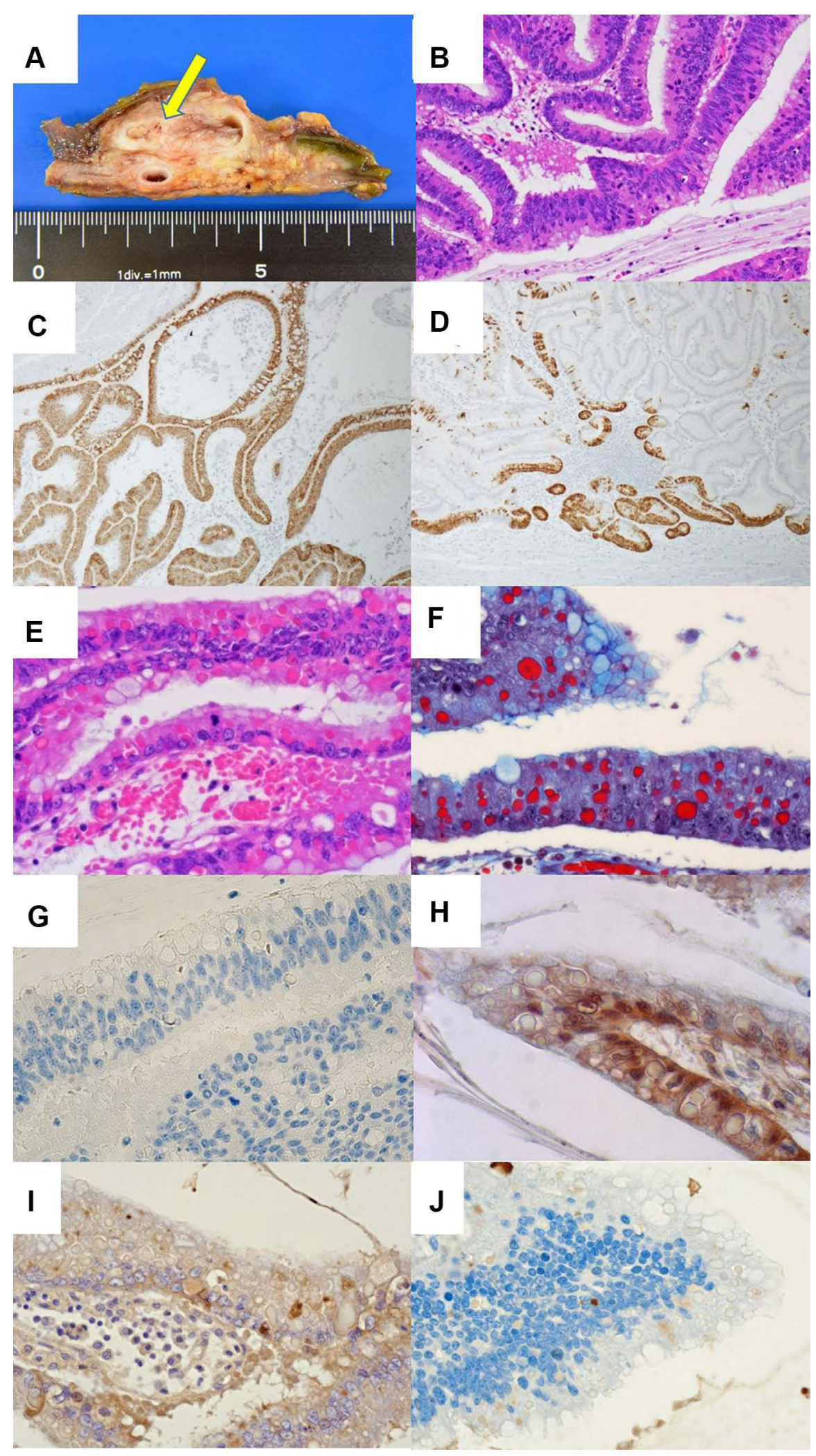

Figure I Case I. (A) Grossly, a mucinous cystic lesion maximally measured $72 \mathrm{~mm}$, while in this figure a cut surface of the cystic and demarcated lesion measuring $30 \mathrm{~mm}$ is shown (arrow). The mural nodule is not included. (B) Nuclear stratification and anisonucleosis are observed (H\&E). (C) The neoplastic epitheliums are positive for MUC5AC, and (D) focal positive for MUC6. (E) Hyaline globules (HGs) are clustered. HGs are round and acidophilic in appearance and perinuclear in location (H\&E). (F) With Masson's trichrome, the HGs show bright red staining with fuchsinophilia (Masson's trichrome). Immunohistochemically, no HGs were positive for MUC6 (G), ubiquitin (H), I-antitrypsin (I), and cleaved caspase-3 (J). 


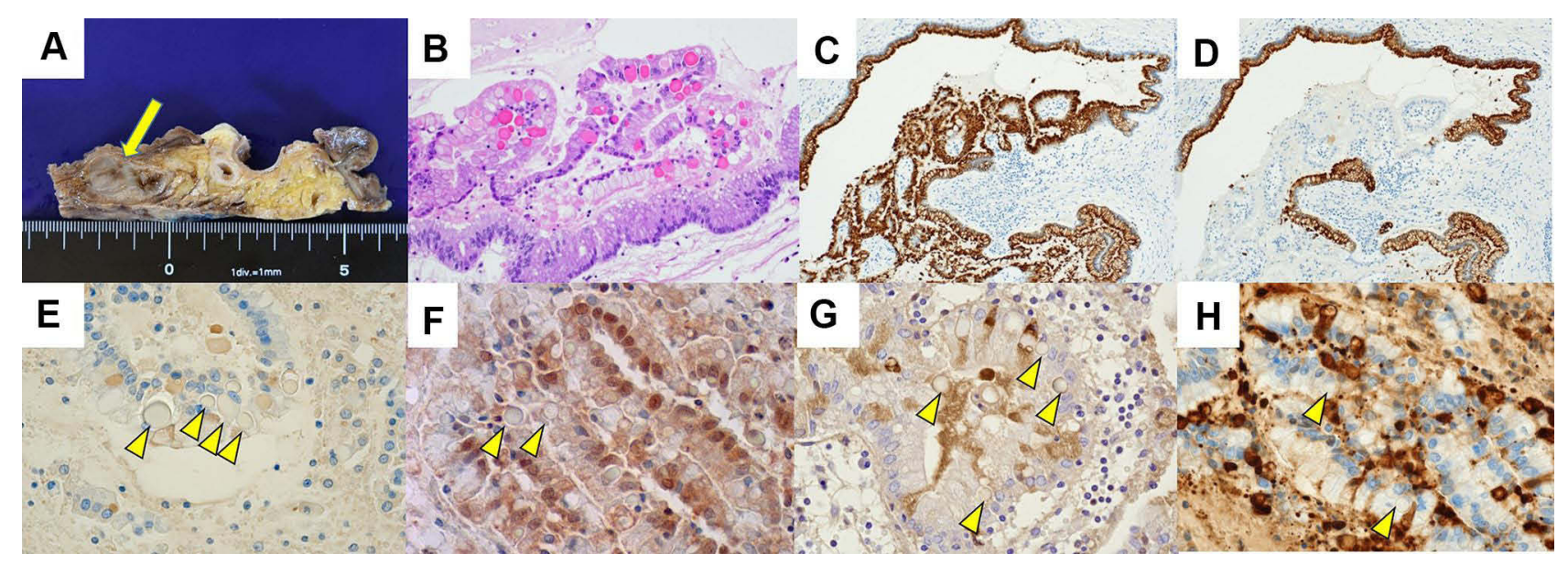

Figure 2 Case 2. (A) Grossly, a mucinous cystic and demarcated lesion, $45 \mathrm{~mm}$ in maximal diameter, is located in the pancreatic parenchyma, while in this figure a cystic and demarcated lesion measuring $25 \mathrm{~mm}$ is demonstrated (arrow). (B) HGs are clustered (H\&E). The neoplastic cells are positive for MUC5AC (C) and MUC6 (D). Immunohistochemically, the HGs were negative for MUC6 (E; arrowheads), ubiquitin (F; arrowheads), I-antitrypsin (G; arrowheads), and cleaved caspase-3 (H; arrowheads).

were processed for ultrastructural observation, as reported previously. ${ }^{6,7}$ The tumor portions with clustered HGs were dug out of the paraffin blocks from two cases of IPMNs with HGs. After deparaffinization overnight, the tissue blocks were dehydrated in graded series of alcohol, refixed overnight at $4 \mathrm{C}$ in $2.5 \%$ glutaraldehyde (Yuai Kasei, Amagasaki, Japan) buffered with $0.1 \mathrm{M}$ sodium cacodylate at $\mathrm{pH} 7.4$, osmified for 2 hours with sodium cacodylatebuffered 1\% osmium tetroxide (Nisshin EM, Tokyo), embedded in epoxy resin (Epon 812, Okenshoji, Tokyo), and polymerized overnight in a $70 \mathrm{C}$ oven. Ultrathin sections were cut using a Diatome diamond (JEOL Japan, Tokyo) at $80 \mathrm{~nm}$ thickness, and stained with uranyl acetate (Ieda Chemicals, Tokyo) and lead citrate (Sigma Aldrich Japan, Tokyo). Images were photographed on a JEOL JEM1400 Flash Electron microscope (JEOL Japan) equipped with an EM-14661FLASH high-sensitivity digital complementary metal-oxide-semiconductor camera.

Case 1: Ultrastructurally, the HGs (or thanatosomes) were round in shape and homogenously electron-dense (Figure $3 \mathrm{~A}-\mathrm{C}$ ). The size of HGs reached $14.5 \mu \mathrm{m}$. The nuclei of the cells with HGs appeared intact without evidence of apoptosis or mitochondria. The apoptotic features were not observed either in the surrounding tumor cells. The gastric-type atypical columnar cells contained apical mucin granules with high electron density.

Case 2: Ultrastructurally, the adenoma cells were rich in mucin production. In addition to clustered electrondense mucin granules, electron-dense, large globular inclusions (HGs) were observed in the supranuclear cytoplasm (Figure 3D and E). The size of the HGs reached $21.6 \mu \mathrm{m}$.
Exocrine-type mucin granules were seen adjacent to the HGs. No apoptotic features or mitochondria were observed in the surrounding tumor cells.

\section{Discussion}

Thanatosomes, a term derived from the Greek words "thanatos" meaning death and "soma" meaning body, are intracellular globular structures that are formed as a consequence of cell turnover. ${ }^{1,2}$ Thanatosomes (or HGs) have been described in neoplastic as well as nonneoplastic diseases in varied tissues and organs. ${ }^{1,2,8-11}$ In H\&E-stained preparations, the HGs typically appear as intracytoplasmic eosinophilic, rounded structures of varying size. HGs homogeneously stain magenta with PAS reaction with diastase-resistance and appear fuchsinophilic with Masson's trichrome preparation. Ultrastructurally, the HGs consisted of large and homogeneously electron-dense or osmiophilic inclusions. Papadimitriou et al described that the HGs ultrastructurally appeared as phagosomes/secondary lysosomes or areas of cytoplasmic condensation surrounded by rough endoplasmic reticulum whorls. ${ }^{1}$ Double stains for apoptosis and plasma proteins confirmed the increased plasma membrane permeability to allow accumulation of proteins in the apoptotic cells to form HGs (Figure 4A). ${ }^{1}$ Pancreatic neoplasms with HGs were associated with oxyphilic cytoplasm containing numerous mitochondria. ${ }^{3}$ In the present ultrastructural study, the HGs seen in two lesions of IPMN represented little evidence of apoptosis or mitochondria. HGs were composed of a homogeneous "proteinaceous" component. The main component of apoptotic bodies should be DNA fragments, and immunohistochemically positive 


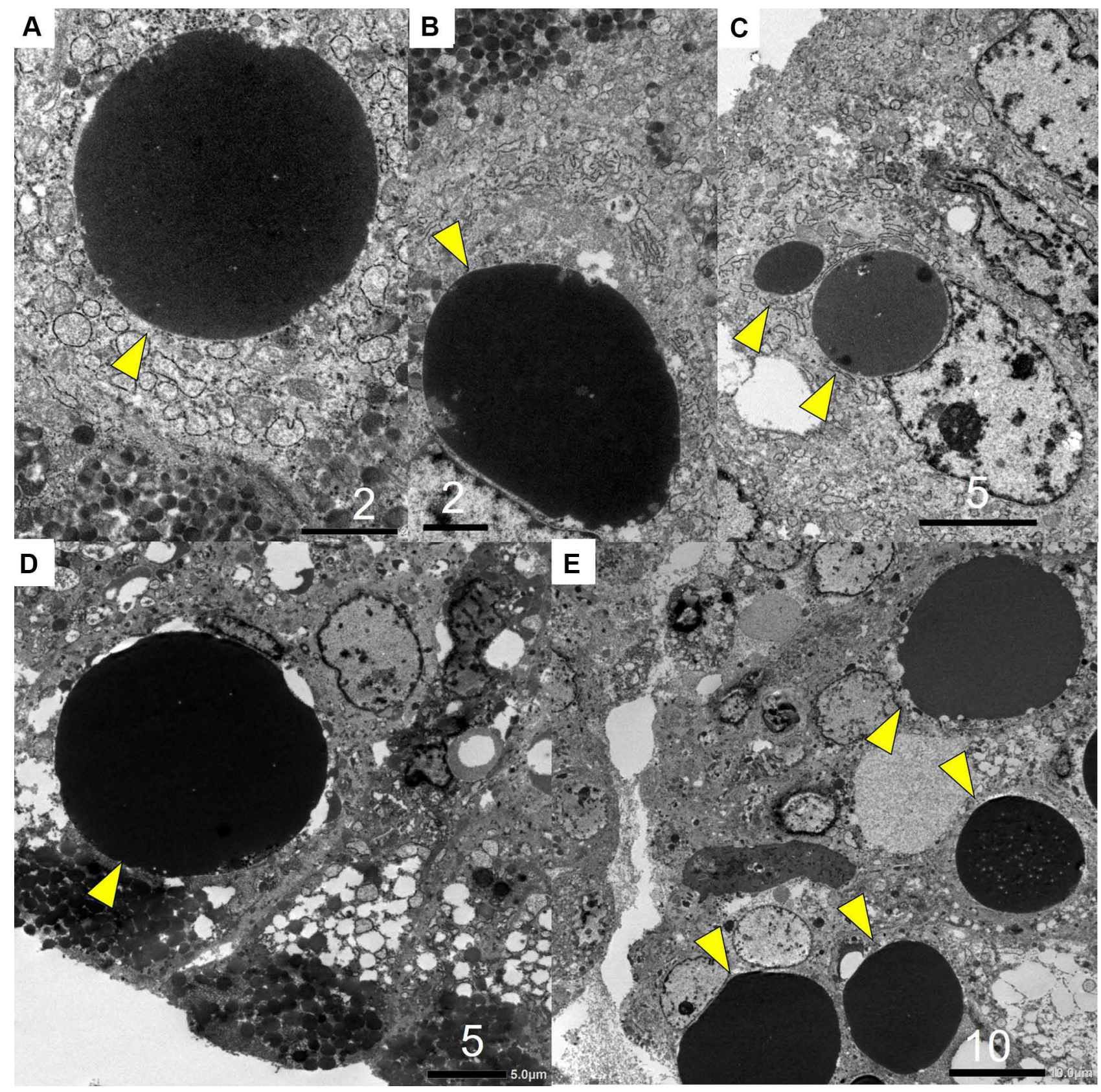

Figure 3 Ultrastructural findings of the HGs in IPMN. (A-C) Ultrastructural findings of the HGs in Casel. The neoplastic cells are rich in mucin production. In addition to clustered electron-dense mucin granules, large-sized and globular electron-dense (osmiophilic) inclusions (HGs or thanatosomes) (arrowheads) are observed in the supranuclear cytoplasm. The size of the HGs reaches $14.5 \mu \mathrm{m}$. [bar $=2 \mu \mathrm{m}$ (a), bar $=2 \mu \mathrm{m}$ (b), bar $=5 \mu \mathrm{m}$ (c)]. (D and E) Ultrastructural findings of the HGs in Case2. The neoplastic cells show prominent mucin production. Large-sized HGs (arrowheads) are observed in the supranuclear cytoplasm. The size of the reaches $21.6 \mu$ m. Exocrine (mucin) granules are seen just adjacent to the HGs. [bar $=5 \mu \mathrm{m}$ (D), bar $=10 \mu \mathrm{m}$ (E)].

for cleaved caspase- $3 .{ }^{12}$ It is expected that mitochondria or nucleic acid components may have unique heterocondensations. We can therefore conclude that the HGs are not related to the apoptotic process but to the condensation of secretory proteinaceous substances in the tumor cells of pancreatic IPMN (Figure 4B). The HGs in the pancreatic intraductal oncocytic papillary neoplasm were extracellular materials in the gland lumens, negative for MUC, 1-antitrypsin. ${ }^{3}$ The HGs in the solid-pseudopapillary neoplasm were intracytoplasmic and extracytoplasmic substances, and the HGs in the clear cell ductal adenocarcinoma were seen within gland lumens and were seen focally within the cytoplasm. ${ }^{4,5}$ As point out earlier, the HGs in the present two cases were intracellular materials, negative for MUC, p53, ubiquitin, 1-antitrypsin, and cleaved caspase-3 

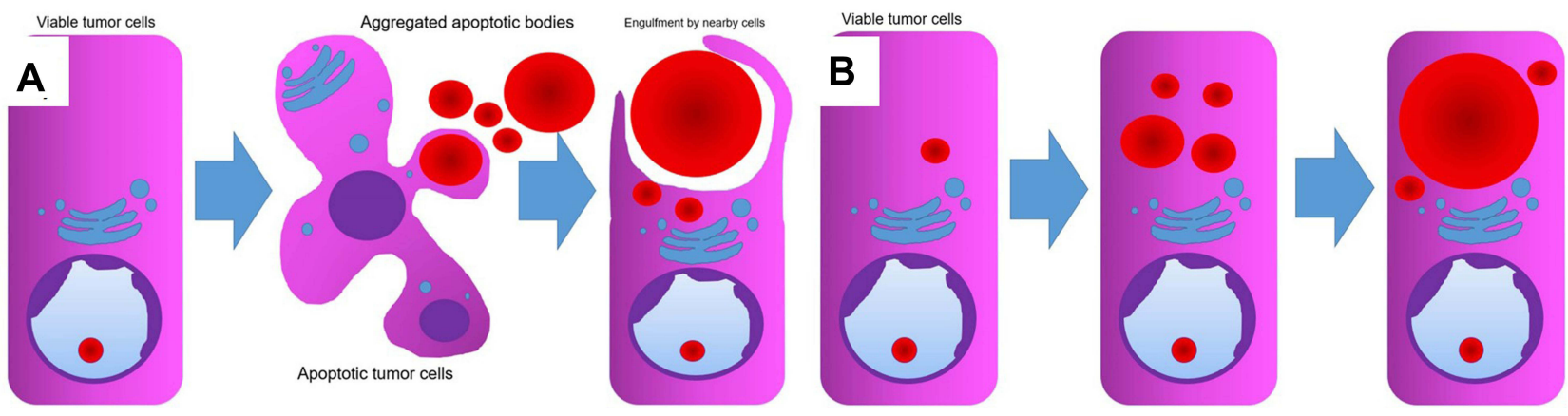

Figure 4 Conventional and our model for the formation of HGs. (A) In conventional model, an intact tumor cell responds to a variety of injurious stimuli through apoptosis. The apoptotic bodies are engulfed by neighboring viable cells. (B) In our model proposed, the HGs are related to the condensation of secretory proteinaceous substances in non-injured tumor cells.

Ubiquitin is an approximately $8.5 \mathrm{kDa}$ protein, comprises 76 amino acids with a high degree of sequence conservation among different species. It is expressed in all types of cells (hence the term ubiquitin) and exists either free or covalently attached (ubiquitinated) as a posttranslational modification to expressed proteins. Once a protein is ubiquitinated in the cell, it can be degraded by the proteasome. ${ }^{13} \mathrm{~A}$ recent study said that the ubiquitination is a widespread post-translational modification that controls multiple steps in autophagy, a major lysosome-mediated intracellular degradation pathway. ${ }^{14}$

In current two cases, the HGs were negative for ubiquitin, immunohistochemically, and there was no evidence that autophagy was occurring, electron microscopically.

We should emphasize the fact that routinely prepared paraffin blocks were consistently available to fine structural evaluations, as was reported previously. ${ }^{6,7}$ To the best of our knowledge, the present study belongs to the first analysis of HGs in pancreatic gastric type IPMN. We suppose that careful microscopic observation may reveal HGs in IPMN more frequently than expected. Further accumulation of cases is needed to clarify the incidence and pathobiological/clinical significance of HGs seen in this type of tumor.

\section{Abbreviations}

CA19-9, carbohydrate antigen 19-9; CDX2, caudal-type homeobox-2; H\&E, hematoxylin and eosin; HG, hyaline globule; IPMN, intraductal papillary mucinous neoplasm; MUC, mucin; PAS, periodic acid-Schiff.

\section{Data Sharing Statement}

The datasets generated during and/or analyzed during the current study are available from the corresponding author on reasonable request.

\section{Patient Consent Statement}

All the procedures were in accordance with the ethical standards of the responsible institutional committee on human experimentation and with the Helsinki Declaration of 1964 and later versions. The patient's husband (case 1) and the patient (case 2) gave a written informed consent to publication as a research document. Case 1 suffers dementia, so that her husband became a proxy.

\section{Acknowledgments}

We cordially thank Kazuhiro Nakajima, M.T., Department of Diagnostic Pathology, Shimada Municipal Hospital, Shimada, Shizuoka, Japan, and Kohei Watanabe, M.T., Special Reference Laboratories, Hamura, Tokyo, Japan, for their skillful technical assistance and secretarial help. The skillful technical assistance by Naoki Ooishi, M.T., and Kuniaki Muramatsu, M.T., Department of Diagnostic Pathology, Shimada Municipal Hospital, Shimada, are also acknowledged. There were no funding sources for reporting the present study. This case was presented at the $110^{\text {th }}$ Annual Meeting of the Japanese Society of Pathology at Tokyo, Japan, 2021.

\section{Author Contributions}

Each author has sufficiently participated in the work to take public responsibility for appropriate portions of the content, and is accountable for the contents of the article. MT performed histopathological diagnosis of the resected samples, analyzed the data, drafted the figure, and made a major contribution to writing the manuscript. RK and KK performed clinical evaluations, surgical treatment and clinical follow-up, and deeply discussed on the clinical significance of the novel microscopic findings. YT analyzed histopathological features and brushed the 
manuscript up. All authors made substantial contributions to conception and design, acquisition of data, or analysis and interpretation of data; took part in drafting the article or revising it critically for important intellectual content; agreed to submit to the current journal; gave final approval of the version to be published; and agree to be accountable for all aspects of the work.

\section{Disclosure}

The authors declare that there is no conflict of interests regarding the publication of the present study.

\section{References}

1. Papadimitriou JC, Drachenberg CB, Brenner DS, Newkirk C, Trump BF, Silverberg SG. "Thanatosomes": a unifying morphogenetic concept for tumor hyaline globules related to apoptosis. Hum Pathol. 2000;31:1455-1465.

2. Dikov DI, Auriault ML, Boivin JF, Sarafian VS, Papadimitriou JC. Hyaline globules (thanatosomes) in gastrointestinal epithelium. Pathophysiologic correlations. Am J Clin Pathol. 2007;127:792-799.

3. Tajiri $\mathrm{T}$, Inagaki $\mathrm{T}$, Ohike $\mathrm{N}$, et al. Intraductal oncocytic papillary carcinoma of the pancreas showing numerous hyaline globules in the lumen. Pathol Int. 2010;60:48-54.

4. Chakhachiro ZI, Zaatari G. Solid-pseudopapillary neoplasm, a pancreatic enigma. Arch Pathol Lab Med. 2009;133:1989-1993.
5. Ray S, Lu Z, Rajendiran S. Clear cell ductal adenocarcinoma pf pancreas, a case report and review of the literature. Arch Pathol Lab Med. 2004;128:693-696.

6. Tsutsumi Y. Electron microscopic study using formalin-fixed, paraffin-embedded material, with special reference to observation of microbial organisms and endocrine granules. Acta Histochem Cytochem. 2018;51:63-71.

7. Tachibana M, Tsutsumi Y. Colitis nucleomigrans: the third type of microscopic colitis (part 2). An ultrastructural study. Pathol Int. 2020;70:761-766.

8. Beranek JT. Thanatosomes and cardiomyocyte apoptotic bodies. Hum Pathol. 2001;32:894-895.

9. Dikov D, Roland J, Chatelet F-P, Cywiner-Golenzer C, Dimitrakov J. Hyaline globules (thanatosomes) in prostate disease. Am J Surg Pathol. 2003;27:700-702.

10. D'Alfonso TM, Ginter PS, Salvatore SP, Antonio LB, Hoda SA. Phylloides tumor with numerous thanatosomes ("death bodies"): a report of two cases and a study of thanatosomes in breast tumors. Int J Surg Pathol. 2014;22:337-342.

11. Panicker NK, Buch AC, Patel AR. Breast carcinoma with numerous large "thanatosomes". J Canr Res Ther. 2015;11:980-982.

12. Tachibana M, Hanaoka T, Watanabe S, Matsushita M, Isono $T$, Tsutsumi Y. Colitis nucleomigrans: the third type of microscopic colitis (part 1). Pathol Int. 2020;70:752-760.

13. Akutsu M, Dikic I, Bremm A. Ubiquitin chain diversity at a glance. $J$ Cell Sci. 2016;129:875-880.

14. Grumati P, Dikic I. Ubiquitin signaling and autophagy. J Biol Chem. 2018;293:5404-5413.

\section{Publish your work in this journal}

The International Medical Case Reports Journal is an international, peer-reviewed open-access journal publishing original case reports from all medical specialties. Previously unpublished medical posters are also accepted relating to any area of clinical or preclinical science. Submissions should not normally exceed 2,000 words or 4 published pages including figures, diagrams and references. The manuscript management system is completely online and includes a very quick and fair peer-review system, which is all easy to use. Visit http://www.dovepress.com/testimonials.php to read real quotes from published authors. 